DFTT-49/2009

LTH 836

\title{
Current Exchanges for Reducible Higher Spin Multiplets and Gauge Fixing
}

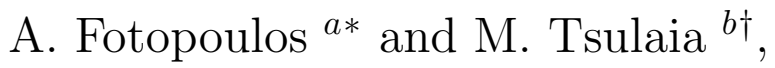 \\ ${ }^{a}$ Dipartimento di Fisica Teorica dell'Università di Torino and INFN Sezione di \\ Torino, via P. Giuria 1, I-10125 Torino, Italy \\ ${ }^{b}$ Department of Mathematical Sciences, University of Liverpool, Peach Street, \\ Liverpool L69 7ZL, United Kingdom
}

\begin{abstract}
We compute the current exchanges between triplets of higher spin fields which describe reducible representations of the Poincare group. Through this computation we can extract the propagator of the reducible higher spin fields which compose the triplet. We show how to decompose the triplet fields into irreducible HS fields which obey Fronsdal equations, and how to compute the current-current interaction for the cubic couplings which appear in [1] using the decomposition into irreducible modes. We compare this result with the same computation using a gauge fixed (Feynman) version of the triplet Lagrangian which allows us to write very simple HS propagators for the triplet fields.
\end{abstract}

*e-mail: foto@to.infn.it

$\dagger^{\dagger}$ e-mail: tsulaia@liv.ac.uk 


\section{Contents}

1 Introduction 1

2 Higher Spin Triplets: Notation and Conventions 3

3 Decomposition of Higher Spin Triplets Into Irreducible Higher Spin Fields and Current-Current Interactions 8

3.1 Decomposition Of $\mathcal{W}$ States In Terms Of Irreducible Modes . . . . 8

3.2 The Current-Current Interaction . . . . . . . . . . . . . . . 14

4 Current Exchanges For Triplets 16

4.1 Gauge Fixing And The Propagator . . . . . . . . . . . . . . . . 16

4.2 Current Exchange and Comparison . . . . . . . . . . . . 18

5 Conclusions 21

6 Acknowledgements 21

A Appendix A: Decoupling of the Lagrangian for Spin 2 and Spin 4 triplet

21

B Appendix B: Lagrangian Normalization for Irreducible Fields

\section{Introduction}

The theory of interacting massless and massive higher spin (HS) fields (see [2][3] for reviews) is attracting growing interest. Until now the consistent interaction vertices for the massless and massive higher spin fields, both on flat and constant curvature backgrounds, have been obtained in frame - like [4] and in metric-like [5]- 20] formulations. However, the studies in this directions are far from being complete. The most challenging problems are to build the complete systematics of the interacting higher spin fields and understand the possible role and connection of these kind of theories with string and M- Theory. One particular problem involves further study of the cubic interactions which have already been constructed in various approaches in order to obtain effective actions which contain interaction terms of an order higher than cubic (see e.g. [21]).

In the present paper we shall follow the covariant BRST formulation of the interacting higher spin fields [12] (see also [8] for the earlier work in this direction). As a first step in this set-up one constructs a BRST charge and the BRST-invariant free Lagrangian (see 22] - 26] for other gauge-invariant descriptions for massive and massless higher spin fields) which describes the propagation of symmetric higher spin modes either on flat or $A d S$ space. As a result of the gauge-invariant formulation, the free Lagrangian contains a number of auxiliary fields. The total system 
of fields is called a "triplet" for the case of reducible massless symmetric representations of Poincare or Anti de Sitter group [27]-[30], or "generalized triplet" for the case of reducible representations of the Poincare group with mixed symmetry [31]. The second step is to make a consistent nonlinear deformation of the quadratic Lagrangian and of the abelian gauge transformations by building the BRST-invariant cubic vertex. The extension of this method to the case of massive higher spin fields is straightforward, the only difference being that one has to use the BRST charge for massive reducible representations of the Poincare group [32]-[33]. An advantage of this approach is that in contrast to the BRST charge describing the propagation of irreducible higher spin modes [35]- 36], the BRST charge for triplets and generalized triplets has a much simpler form. This in turn simplifies the problem of finding the BRST-invariant cubic interaction vertex for either massless or massive fields.

However, it is not clear how far one can pursue the study of interactions between reducible representations in order to build complete systematics or at least to achieve a better understanding of interacting higher spin theory. Therefore, a study of interactions between the fields which belong to the irreducible representations of Poincare and $A d S$ groups are of extreme importance. There are several reasons for this: apart from the fact that the original interacting higher spin theory has been constructed for irreducible modes in the frame-like approach [4], a BRST charge describing a massive triplet or a generalized triplet on an $A d S$ space has not been constructed yetf. Furthermore, a computation of four point scattering amplitudes, or Witten diagrams in the case of an $A d S$ space for reducible higher spin modes can be rather complicated because of the presence of nonphysical pure gauge degrees of freedom in corresponding Lagrangians. Obviously these degrees of freedom should be gauged away in order to build a consistent perturbation theory. In other words, the pure gauge degrees of freedom, which simplify the structure of the free Lagrangian and the interaction vertexes, cause difficulties when analyzing Feynman diagrams and scattering amplitudes and the main goal of the present paper is to address this problem.

To summarise: our strategy is to start from the Lagrangian describing interacting reducible representations of the higher spin modes, since the construction of these kind of gauge-invariant Lagrangians is much simpler than for irreducible ones. As a second step in order to build the perturbation theory we extract the corresponding propagators for irreducible higher spin modes from the Lagrangian describing massless reducible higher spin fields. This program turns out to be technically rather complicated and we describe it in great detail. As an application of this procedure we consider the problem of current-current exchange for the case where reducible higher spin modes are coupled to scalar fields. We leave the application of the technique developed in this paper for the case of more complicated systems when one has interactions between infinite number of triplets, for further study.

We would like to point out that our results described in Section 3 for diago-

\footnotetext{
${ }^{\ddagger}$ One can construct, however, the Lagrangian describing an interaction between a higher spin modes and massive scalars from the one describing the massless fields [1]
} 
naliziation of the Lagrangian which contains reducible fields in terms of Fronsdal Lagrangians for irreducible fields, has been checked explicitly only up to spin 4 . Nevertheless we have provided an ansatz which we believe that it diagonalizes the triplet Lagrangian for arbitrary spin-s. Based on the non-trivial nature of the diagonalization procedure for spin 4 as well as some qualitative features, which appear in the comparison of these results with those of Section 4 , we suggest that the procedure proposed for the decomposition of the Lagrangian describing "triplets" into the Fronsdal Lagrangians for irreducible fields is correct.

\section{Higher Spin Triplets: Notation and Conven- tions}

In this Section we shall briefly summarise the technique of building BRST-invariant cubic vertexes [12. We shall explain it on an example of massive triplets which, despite being a very simple generalization of the vertex given in [14] has not been presented elsewhere $\S$.

Let us start from the massless triplet in $\mathcal{D}+1$ space-time dimensions. To this end we introduce an auxiliary Fock space spanned by oscillator and ghost variables

$$
\left[\alpha_{M}, \alpha_{N}^{+}\right]=\eta_{M N}, \quad\left\{c^{+}, b\right\}=\left\{c, b^{+}\right\}=\left\{c_{0}, b_{0}\right\}=1
$$

and the vacuum in the Hilbert space is defined as:

$$
\alpha_{M}|0\rangle=0, \quad c|0\rangle=0, \quad b|0\rangle=0, \quad b_{0}|0\rangle=0 .
$$

Obviously, one can consider an arbitrary number of these oscillators, thus describing reducible representations of the Poincare group with mixed symmetry [31]. Although the generalization to this case is straightforward we shall consider only totally symmetric representations. The corresponding BRST charge has the form:

$$
Q=c_{0} \tilde{l}_{0}+c^{+} \tilde{l}+c \tilde{l}^{+}-c^{+} c b_{0}
$$

with $\tilde{l}_{0}=p^{M} p_{M}, \tilde{l}=\alpha^{M} p_{M}, p_{M}=-i \partial_{M}$.

The functional (named "triplet" [28]) which contains both physical reducible representations of the Poincare group with arbitrary integer spins and auxiliary nonphysical fields is the most general expansion in terms of the ghost variables with the ghost number zero

$$
|\Phi\rangle=\left|\phi_{1}\right\rangle+c_{0}\left|\phi_{2}\right\rangle=|\varphi\rangle+c^{+} b^{+}|d\rangle+c_{0} b^{+}|c\rangle
$$

\footnotetext{
$\S$ A related discussion with respect to the high energy limit of Open String Field Theory appears in 15
} 
and the component fields are given by:

$$
\begin{aligned}
|\varphi\rangle & =\frac{1}{s !} \varphi_{M_{1} \ldots M_{s}}(x) \alpha^{M_{1}+} \ldots \alpha^{M_{s}+}|0\rangle \\
|d\rangle & =\frac{1}{(s-2) !} D_{M_{1} \ldots M_{s-2}}(x) \alpha^{M_{1}+} \ldots \alpha^{M_{s-2}+}|0\rangle \\
|c\rangle & =\frac{-i}{(s-1) !} C_{M_{1} \ldots M_{s-1}}(x) \alpha^{M_{1}+} \ldots \alpha^{M_{s-1}+}|0\rangle .
\end{aligned}
$$

Furthermore, one can perform a dimensional reduction to $\mathcal{D}$ dimensions thus describing a massive theory in one dimension lower [32]-33]. The corresponding BRST charge

$$
Q=c_{0} l_{0}+c^{+} l+c l^{+}+c_{0} m^{2}+c^{+} m \alpha_{D}+c m \alpha_{D}^{+}-c^{+} c b_{0}, \quad l_{0}=p^{\mu} p_{\mu}, \quad l=\alpha^{\mu} p_{\mu}
$$

contains the constant mass parameter $m$ and therefore all fields in the triplet have the same value of mass.However one can make the mass parameter oscillator dependent [34] thus considering a Regge trajectory similar to the one present in the bosonic string theory. The construction of the interaction vertex in this case is much more involved and we shall not consider this interesting possibility here. Having constructed the nilpotent BRST charge one can write the BRST-invariant free Lagrangian

$$
\mathcal{L}=\int d c_{0}\langle\Phi|Q| \Phi\rangle
$$

which is invariant under the gauge transformations

$$
\delta|\Phi\rangle=Q|\Lambda\rangle, \quad|\Lambda\rangle=b^{+}|\lambda\rangle, \quad|\lambda\rangle=\frac{i}{(s-1) !} \lambda_{M_{1} \ldots M_{s-1}}(x) \alpha^{M_{1}+} \ldots \alpha^{M_{s-1}+}|0\rangle
$$

The free equations of motion and gauge transformation rules for the massive triplet can be easily obtained from (2.4), (2.5), (2.6) and (2.7) after making the decomposition $\alpha_{M}^{+} \rightarrow\left(\alpha_{\mu}^{+}, \alpha_{D}^{+}\right)$

$$
\begin{gathered}
\left(l_{0}+m^{2}\right)|\varphi\rangle=\left(l^{+}+m \alpha_{D}^{+}\right)|c\rangle \\
\left(l_{0}+m^{2}\right)|d\rangle=\left(l+m \alpha_{D}\right)|c\rangle \\
|c\rangle=\left(l^{+}+m \alpha_{D}^{+}\right)|d\rangle-\left(l+m \alpha_{D}\right)|\varphi\rangle
\end{gathered}
$$

while the gauge transformation rule (2.7) gives

$$
\delta|\varphi\rangle=\left(l^{+}+m \alpha_{D}^{+}\right)|\lambda\rangle, \quad \delta|d\rangle=\left(l+m \alpha_{D}\right)|\lambda\rangle, \quad \delta|c\rangle=\left(l_{0}+m^{2}\right)|\lambda\rangle .
$$

In order to describe cubic interactions one introduces three copies $(i=1,2,3)$ of the Hilbert space defined above, as in bosonic Open String Field Theory [37]. Then the Lagrangian has the form

$$
\mathcal{L}=\sum_{i=1}^{3} \int d c_{0}^{i}\left\langle\Phi_{i}\left|Q_{i}\right| \Phi_{i}\right\rangle+g\left(\int d c_{0}^{1} d c_{0}^{2} d c_{0}^{3}\left\langle\Phi_{1}\right|\left\langle\Phi_{2}\right|\left\langle\Phi_{3}|| V\right\rangle+h . c\right),
$$


where $|V\rangle$ is the cubic vertex and $g$ is a coupling constant. The Lagrangian (2.12) is invariant up to the first order in the coupling constant $g$ with respect to the nonabelian gauge transformations

$$
\delta\left|\Phi_{i}\right\rangle=Q_{i}\left|\Lambda_{i}\right\rangle-g \int d c_{0}^{i+1} d c_{0}^{i+2}\left[\left(\left\langle\Phi_{i+1}\right|\left\langle\Lambda_{i+2}\right|+\left\langle\Phi_{i+2}\left|\left\langle\Lambda_{i+1}\right|\right) \mid V\right\rangle\right],\right.
$$

provided that the vertex $|V\rangle$ satisfies the BRST invariance condition

$$
\sum_{i} Q_{i}|V\rangle=0
$$

Further on, in order to simplify equations in the rest of this section we introduce bilinear combinations of the oscillators

$$
\gamma^{+, i j}=c^{+, i} b^{+, j}, \quad \beta^{+, i j}=c^{+, i} b_{0}^{j} \quad M^{+, i j}=\frac{1}{2} \alpha^{+, \mu, i} \alpha^{+, \mu, j}
$$

which have ghost number zero. Further we take an ansats for the vertex

$$
\begin{gathered}
|V\rangle=V^{1} \times V^{2}|-\rangle_{123}, \\
|-\rangle_{123}=c_{0}^{1} c_{0}^{2} c_{0}^{3}|0\rangle_{1} \otimes|0\rangle_{2} \otimes|0\rangle_{3}
\end{gathered}
$$

with

$$
\begin{gathered}
V^{1}=\exp \left(Y_{i j} l^{+, i j}+Z_{i j} \beta^{+, i j}+U_{i j} m^{i} \alpha_{D}^{+, j}\right), \\
V^{2}=\exp \left(S_{i j} \gamma^{+, i j}+P_{i j} M^{+, i j}+R_{i j} \alpha_{D}^{+, i} \alpha_{D}^{+, j}\right),
\end{gathered}
$$

where $P_{i j}=P_{j i}, R_{i j}=R_{j i}$. We have also assumed that $m_{1}=m_{2}=m_{3}$. However, this requirement is not a necessity and one can still find a solution when this requirement is relaxed. Putting this ansatz into the BRST invariance condition and using momentum conservation $p_{\mu}^{1}+p_{\mu}^{2}+p_{\mu}^{3}=0$ one can obtain a solution for $Y^{r s}, U^{r s}$ and $Z^{\text {rs }}$

$$
\begin{gathered}
Z_{i, i+1}+Z_{i, i+2}=0 \\
Y_{i, i+1}=Y_{i i}-Z_{i i}-1 / 2\left(Z_{i, i+1}-Z_{i, i+2}\right) \\
Y_{i, i+2}=Y_{i i}-Z_{i i}+1 / 2\left(Z_{i, i+1}-Z_{i, i+2}\right) . \\
Z_{i, i}+Z_{i, i+1}+Z_{i, i+2}=U_{i, i}+U_{i+1, i}+U_{i+2, i} \\
S_{i j}=P_{i j}=R_{i j}=0 \quad i \neq j \\
R_{i j}-S_{i i}=0 \quad i=1,2,3 \\
P_{i i}-S_{i i}=0 \quad i=1,2,3
\end{gathered}
$$

In what follows we will assume cyclic symmetry in the three Fock spaces which implies along with (2.18) and

$$
Z_{12}=Z_{23}=Z_{31}=Z_{a}, \quad Z_{21}=Z_{13}=Z_{32}=Z_{b}=-Z_{a}
$$




$$
\begin{gathered}
U_{12}=U_{23}=U_{31}=U_{a}, \quad U_{21}=U_{13}=U_{32}=U_{b} \\
Y_{12}=Y_{23}=Y_{31}=Y_{a}, \quad Y_{21}=Y_{13}=Y_{32}=Y_{b} \\
Y_{i i}=Y, \quad Z_{i i}=Z, \quad P_{i i}=P, \quad S_{i i}=S, \quad R_{i i}=R, \\
S=P=R
\end{gathered}
$$

Choosing the value of the parameter $S$ to be equal to 1 one can make the above solution exact to all orders in the coupling constant in complete analogy with [14]. It can be checked directly that this solution belongs to nontrivial cohomologies of the BRST charge (2.5) and thus can not be obtained via the field redefinitions from the free Lagrangian Let us also note that one can make the triplet matrix valued, and consider a theory with a nonabelian gauge group in complete analogy with the string theory.

As an alternative example one can consider a different condition on the mass parameters; in particular $m_{1}+m_{2}+m_{3}=0$. This case will correspond to a dimensional reduction of the vertex given in [14]. In this case parameters $U_{i j}$ will obey exactly the same conditions as the parameters $Y_{i j}$.

An important point is that one can consistently put the mass parameter(s) $m_{i}$ equal to zero and decouple oscillators $\alpha_{D}^{+}$which correspond to the compact dimension. In this way one recovers the interacting system of massless triplets described in [14]. As for the case of interacting massless triplets, the case of interacting massive triplets requires an infinite number of them in order to ensure the exactness of the vertex in all orders in coupling constant $g$.

The next natural step is to construct the full perturbation theory for this model of interacting higher spins. To this end one needs to gauge fix the action in order to avoid propagation of the pure gauge degrees of freedom and extract the propagators of individual physical higher spin modes. This program turns out to be technically involved and as a first step instead of building the perturbation theory for this model, we shall consider a system of interacting triplets where their number is finite. As we mentioned above in this case the vertex is no longer exact to all orders in the coupling constant and therefore there is a certain freedom in the definition of the coupling constants. These coupling constants can be presumably fixed in the full interacting theory which in principle can be different from the solution described above, since one can not claim that this solution is unique.

I The nontrivial cohomology means that the vertex cannot be written in the form $|V\rangle=\tilde{Q}|W\rangle$, where $|W\rangle$ an arbitrary functional having ghost number -2 (see [12] for details). Such interactions are generated by field redefinitions of the form $\left|\varphi_{i}\right\rangle=\left\langle\varphi_{i+1}, \varphi_{i+2}|| W\right\rangle$. Actually if we drop the requirement for cyclic symmetry the most general field redefinition would generate an interaction of the form $|\delta V\rangle=Q_{1}\left|W_{1}\right\rangle+Q_{2}\left|W_{2}\right\rangle+Q_{3}\left|W_{3}\right\rangle$, which corresponds to the case when we redefine the three fields separately in the free Lagrangian using three independent functionals $\left|W_{i}\right\rangle$. Therefore in practice when the cyclic symmetry of the vertex is not required in order to exclude all vertexes which can be obtained from the free Lagrangian via the field redefinitions one needs to check that the solution of cohomologies of the BRST charge $\tilde{Q}$ does not have the form $Q_{1}\left|W_{1}\right\rangle+Q_{2}\left|W_{2}\right\rangle+Q_{3}\left|W_{3}\right\rangle$. 
Therefore in the following, let us consider the simple case of the system of one massless triplet which describes spins $s, s-2, \ldots, 1 / 0$ interacting with two scalar fields [1]. The general solution for a gauge invariant Lagrangian to the lowest order in $g$ can in principle be deduced from (2.18) if one drops the requirement for cyclic symmetry and sets $m_{i}=0$. The exactness of the vertex to all orders in $g$ is also no longer required, so one can consider a finite number of interacting triplets. If we keep the masses of the two scalars nonzero the interaction vertex remains the same as for the massless case, but the free Lagrangian is that of massive scalar triplets. This is the equivalent model to the one considered in [19], where the scalars play the role of matter charged under HS gauge fields. These higher spin fields gauge the rigid symmetries of the free action for the scalars. One can also make a deformation of this solution for an $A d S$ space but we shall not consider this possibility here. In components the free part of the Lagrangian of (2.12), for a spin-s triplet can be written

$$
\begin{aligned}
\mathcal{L} & =-\frac{1}{2}\left(\partial_{\mu} \varphi\right)^{2}+s \partial \cdot \varphi C+s(s-1) \partial \cdot C D \\
& +\frac{s(s-1)}{2}\left(\partial_{\mu} D\right)^{2}-\frac{s}{2} C^{2}
\end{aligned}
$$

where we have rescaled all fields of the triplet by a factor $\varphi_{s} \rightarrow \sqrt{s !} \varphi_{s}$ as can be easily seen by computing the bracket $\langle\Phi|Q| \Phi\rangle$ using the definitions of (2.4). The free equations of motion for a triplet on a flat background are

$$
\begin{aligned}
& \square \varphi=\partial C, \\
& C=\partial \cdot \varphi-\partial D, \\
& \square D=\partial \cdot C,
\end{aligned}
$$

along with the gauge transformations

$$
\delta \phi=\partial \lambda, \quad \delta C=\square \lambda, \quad \delta D=\partial \cdot \lambda .
$$

Here, as usual, $\partial$. denotes the divergence and $\partial$ denotes the symmetrized derivative without contraction of indexes. Using the expression of the vertex in [1, or equivalently setting $m_{i}=0$ and dropping the cyclicity requirement in (2.16)-(2.21), we can write the cubic interaction for two scalars and one arbitrary HS triplet

$$
\mathcal{L}_{\text {int }}^{00 s}=\sum_{q=0}^{\left[\frac{s}{2}\right]} \frac{N_{s-2 q}}{(2 q) ! !(s-2 q) !} \mathcal{W}_{s}^{q} \cdot J_{s-2 q}^{1 ; 2}+\text { h.c. },
$$

where $\mathcal{W}_{s}^{q}$ is defined in [28]

$$
\mathcal{W}_{s}^{q}=\varphi_{s}^{[q]}-2 q D_{s-2}^{[q-1]}, \quad \delta \mathcal{W}_{s}^{q}=\partial \Lambda_{s-1}^{[q]},
$$


and $\varphi_{s}^{[q]}$ is the qth trace of the tensors $\varphi_{s}$ of rank-s. The currents are defined as [7]

$$
J_{s-2 q}^{1 ; 2}=\sum_{r=0}^{s-2 q}\left(\begin{array}{c}
s-2 q \\
r
\end{array}\right)(-1)^{r}\left(\partial^{\mu_{1}} \ldots \partial^{\mu_{r}} \phi_{1}\right)\left(\partial^{\mu_{r+1}} \ldots \partial^{\mu_{s-2 q}} \phi_{2}\right)
$$

and $N_{s-2 q}$ is an undetermined constant which probably gets fixed once we have the fully consistent, interacting HS theory to all orders in the coupling constant. That is, we expect that closing the algebra of gauge transformations and gauge invariance of higher order interactions will constrain these coefficients. Their precise values are not important for our present discussion and we will not use them any more, but we will assume that they are non-vanishing and therefore consistent interactions of the type in (2.25) do exist in the fully-gauge invariant theory. Moreover the explicit form of the currents is not needed in what follows. Since we shall consider current-current interactions for external currents with intermediate HS states propagating, the only property we shall use for our computations is their conservation. Nevertheless, if one considers a scattering process between dynamical scalar fields, the explicit form of the currents is needed [19].

Our goal in the following sections is to compute the current-current interaction between the currents (2.27) using two methods. First, by decomposing $\mathcal{W}_{s}^{q}$ into irreducible fields; and second by directly using the $\mathcal{W}_{s}^{q}$ propagator in a particular gauge. This way we will deduce the propagator of the triplet fields in (2.22) which to our knowledge has not been considered elsewhere.

\section{Decomposition of Higher Spin Triplets Into Irreducible Higher Spin Fields and Current- Current Interactions}

In order to compute scattering amplitudes using Feynman rules we will need to decompose the triplets into irreducible modes and apply this decomposition to the free Lagrangian (2.22). We expect that the Lagrangian for the triplet will become a sum of Fronsdal Lagrangians for irreducible higher spin states of spin $s, s-2, \ldots, 1 / 0$. This decomposition is a long and difficult task which to our knowledge has not been presented elsewhere. We will take an interacting Lagrangian to be of the form (2.25) with currents given by linear combinations of those in (2.27). Once we have completed this task we will compute the current exchanges using the same methods as in [13].

\subsection{Decomposition Of $\mathcal{W}$ States In Terms Of Irreducible Modes}

In this subsection we will demonstrate how we can decompose the fields $\mathcal{W}_{s}^{q}$ in terms of individual (Fronsdal) higher spin modes $\Psi$. We use the $\mathcal{W}_{s}^{q}$ fields since 
their gauge transformation has the simple form (2.26). Nevertheless, $\mathcal{W}$ transform with a tracefull gauge parameter and off shell their double trace is not zero, unlike the double trace of the irreducible higher spin mode which appears in the Fronsdal description [5]. Moreover, the equations of motion for $\mathcal{W}_{s}^{q}$ are not decoupled among each other

$$
\mathcal{F} \mathcal{W}_{s}^{q}=\square \mathcal{W}_{s}^{q}-\partial \partial \cdot \mathcal{W}_{s}^{q}+\partial^{2}\left(\mathcal{W}_{s}^{q}\right)^{\prime}=\partial^{2} \mathcal{W}_{s}^{q+1}
$$

where $\mathcal{F}$ is the Fronsdal operator. After complete gauge fixing the triplet describes irreducible HS fields with spins $s, s-2 \ldots 1 / 0$. These physical modes correspond to the on-shell modes of $\mathcal{W}_{s}^{q}[28,3]$. Notice that setting in (3.1) $\mathcal{W}_{s}^{q}=0, q \geq 1$ we recover the Fronsdal equations of motion for an irreducible HS field of spin-s and all lowest spin fields effectively dissapear. Our goal is to extract irreducible Fronsdal fields from the triplet Lagrangian, which are double traceless (off shell) and transform with a traceless gauge parameter.

Let us demonstrate with a few low spin examples our decomposition method and then we will give the general formula. It is more convenient to work with the Lagrangian after we have eliminated the auxiliary field $C_{s-1}$

$$
\begin{aligned}
\mathcal{L} & =-\frac{1}{2}\left(\partial_{\mu} \varphi\right)^{2}+\frac{s}{2}(\partial \cdot \varphi)^{2}+s(s-1) \partial \cdot \partial \cdot \varphi D \\
& +s(s-1)\left(\partial_{\mu} D\right)^{2}+\frac{s(s-1)(s-2)}{2}(\partial \cdot D)^{2} .
\end{aligned}
$$

Let us note, that the non dynamical field $C$ naturally appears as a result of a general expansion of the functional $|\Phi\rangle$ in terms of the ghost variables. Moreover its presence is required by the form of the gauge transformations (2.7) since the BRST charge (2.3) when acting on the parameter of gauge transformations $|\Lambda\rangle$ gives rise to a term $-c_{0} b^{+} \square|\lambda\rangle$ proportional to the combination $c_{0} b^{+}$. In principle one can consider the system with the constrained parameter of gauge transformations $\square \lambda=0$. Otherwise one can notice that the gauge transformation rule for the field $C$ coincides with the one for $\partial \cdot \varphi-\partial D$. Therefore one can express the field $C$ in terms of the fields $\varphi$ and $D$ before constructing the BRST invariant Lagrangian and thus consider a "doublet" formulation for the free reducible higher spin modes. However in a case of interacting triplets, depending on a particular vertex under consideration it is not so easy in general to guess a correct form of the expression of fields $C_{i}$ in terms of the fields $\varphi_{i}$ and $D_{i}$. Although in the particular example of a cubic interaction considered in the present paper one can still do so (the gauge transformation rule for $\phi, C$ and $D$ fields which describe the higher spin triplet does not change, whereas the scalars do not bring about $C$ fields [1]) we keep the field $C$ from the beginning in order to keep a systematic BRST approach for interacting triplets.

The Lagrangian above can be written in a fully symmetrized form

$$
\begin{aligned}
\mathcal{L} & =-\frac{1}{2(s+1)}(\partial \varphi)^{2}+s(\partial \cdot \varphi)^{2}-s(\partial \cdot \varphi)(\partial D) \\
& +s(\partial D)^{2}-\frac{s(s-1)(s-2)}{2}(\partial \cdot D)^{2}
\end{aligned}
$$


where we have used the following identity for any symmetric field of spin s

$$
\left(\partial_{\mu} \varphi\right)^{2}=\frac{1}{s+1}(\partial \varphi)^{2}-s(\partial \cdot \varphi)^{2} .
$$

We will also use the following identities and conventions described in [28]

$$
\begin{aligned}
& \left(\partial^{p} \varphi\right)^{\prime}=\square \partial^{p-2} \varphi+2 \partial^{p-1} \partial \cdot \varphi+\partial^{p} \varphi^{\prime}, \\
& \partial^{p} \partial^{q}=\left(\begin{array}{c}
p+q \\
p
\end{array}\right) \partial^{p+q}, \\
& \partial \cdot\left(\partial^{p} \varphi\right)=\square \partial^{p-1} \varphi+\partial^{p} \partial \cdot \varphi, \\
& \partial \cdot \eta^{k}=\partial \eta^{k-1}, \\
& \left(\eta^{k} T_{(s)}\right)^{\prime}=[d+2(s+k-1)] \eta^{k-1} T_{(s)}+\eta^{k} T_{(s)}^{\prime},
\end{aligned}
$$

The symmetrization notation is the one of [28]. Finally, for completeness we give the Fronsdal Lagrangian [5] for an irreducible higher spin field $\Psi$

$$
\begin{aligned}
\mathcal{L}= & -\frac{1}{2}\left(\partial_{\mu} \Psi\right)^{2}+\frac{s(s-1)}{4}\left(\partial_{\mu} \Psi^{\prime}\right)^{2}+\frac{s}{2}(\partial \cdot \Psi)^{2} \\
& +\frac{s(s-1)}{2} \Psi^{\prime}(\partial \cdot \partial \cdot \Psi)+\frac{s(s-1)(s-2)}{8}\left(\partial \cdot \Psi^{\prime}\right)(\partial \cdot \Psi)
\end{aligned}
$$

Spin-2 We make the ansatz

$$
\begin{aligned}
& \mathcal{W}_{2}^{0}=\varphi_{\mu \nu}=\Psi_{\mu \nu}-A \eta_{\mu \nu} \Psi \\
& \mathcal{W}_{2}^{1}=\varphi^{\prime}-2 D=\Psi
\end{aligned}
$$

By direct substitution in (3.2) we can easily see that the $\Psi_{\mu \nu}, \Psi$ fields decouple for $A=-\frac{1}{d-2}$ and the Lagrangian becomes

$$
\mathcal{L}=-\frac{1}{2}\left(\partial_{\mu} \Psi_{\rho \sigma}\right)^{2}+\left(\partial_{\nu} \Psi_{\mu}^{\nu}\right)^{2}+\Psi^{\prime} \partial_{\mu} \partial_{\nu} \Psi^{\mu \nu}+\frac{1}{2}\left(\partial_{\mu} \Psi^{\prime}\right)^{2}-\frac{1}{2(d-2)}\left(\partial_{\mu} \Psi\right)^{2} .
$$

The Lagrangian above describes a massless spin-2 particle and a scalar. We note that the normalization of the kinetic term of the scalar is not canonical and this will have to be taken into account when computing the current exchanges since the residue of the propagator will not be the standard one. Now we can solve (3.7) for $\Psi_{\mu \nu}$ and $\Psi$ in terms of the fields $\mathcal{W}_{2 ; \mu \nu}^{0}$ and $\mathcal{W}_{2}^{1}$ the solutions is

$$
\begin{aligned}
\Psi_{\mu \nu} & =\mathcal{W}_{2 ; \mu \nu}^{0}-\frac{1}{d-2} \eta_{\mu \nu} \mathcal{W}_{2}^{1} \\
\Psi & =\mathcal{W}_{2}^{1} .
\end{aligned}
$$


Spin-4 The above spin-2 result suggests that we expand the $\Psi_{n}$ fields in terms of $\mathcal{W}_{s}^{q}$. This is a crucial observation based on the fact that: i) $\mathcal{W}_{s}^{q}$ transform like irreducible modes but with traceful parameters of gauge transformations ii) the fields $\Psi_{n}$, since they are irreducible, should be double traceless $\Psi_{n}^{\prime \prime}=0, n>3$, and should transform with traceless gauge parameter. We make the ansatz

$$
\begin{aligned}
\Psi_{4} & =\mathcal{W}_{4}^{0}+A \eta \mathcal{W}_{4}^{1}+B \eta^{2} \mathcal{W}_{4}^{2} \\
\Psi_{2} & =\mathcal{W}_{4}^{1}+C \eta \mathcal{W}_{4}^{2} \\
\Psi_{0} & =\mathcal{W}_{4}^{2}
\end{aligned}
$$

where $\Psi_{n}$ denotes irreducible higher spin modes with spin $n$. From (3.10) and (2.26) one can see that, the gauge transformation rule for the field $\Psi_{4}$ is $\delta \Psi_{4}=\partial \tilde{\Lambda}_{3}$ with $\tilde{\Lambda}_{3}$ given by

$$
\tilde{\Lambda}_{3}=\Lambda_{3}+A \eta \Lambda_{3}^{\prime} .
$$

If we demand that $\tilde{\Lambda}_{3}$ is a traceless tensor of the third rank, as required by [5] we get $A=-\frac{1}{d+2}$. However the requirement of the "proper" gauge transformation does not fix all coefficients in (3.10). In order to fix the remaining coefficients one uses the condition $\Psi_{4}^{\prime \prime}=0$ and

$$
\left(\mathcal{W}^{q}\right)^{\prime}=\frac{1}{q+1} \varphi^{[q+1]}+\frac{q}{q+1} \mathcal{W}^{q+1}
$$

to obtain

$$
A=-\frac{1}{d+2} \quad B=\frac{1}{d(d+2)}
$$

So the double tracelessness condition allows us to fix most of the coefficients in (3.10) and moreover guarantees that $\Psi_{4}$ transforms as an irreducible field of spin 4 in agreement with [5]. We note that we could have tried to put a $\mathcal{W}^{0^{\prime}}$ term in the expansion of $\Psi_{2}$ but this would give gauge transformation terms of the form $\partial \cdot \Lambda_{3}$ which are not appropriate for an irreducible mode. The remaining coefficients in (3.10) cannot be fixed by the double tracelessness condition. We assume that the decomposition of $\Psi_{2}, \Psi_{0}$ in terms of $\mathcal{W}_{4}^{1}$ and $\mathcal{W}_{4}^{2}$ is the same as the decomposition of the spin-2 triplet in (3.9), that is we set $C=-\frac{1}{d+2}$. This assumption will be verified later on when we will demonstrate that this choice leads to a complete decoupling of the $\Psi_{n}$ fields in the free Lagrangian. Of course we can solve the equations above for $\mathcal{W}$ or for $\varphi, D$ in terms of for $\Psi_{4}, \Psi_{2}$ and $\Psi$. The resulting expressions are (the subscript in $\Psi_{n}$ denotes the rank of the symmetric tensor field):

$$
\begin{aligned}
& \mathcal{W}_{4}^{0}=\Psi_{4}+\frac{1}{d+2} \eta \Psi_{2}+\frac{1}{d(d-2)} \eta^{2} \Psi_{0} \\
& \mathcal{W}_{4}^{1}=\Psi_{2}+\frac{1}{d-2} \eta \Psi_{0} \\
& \mathcal{W}_{4}^{2}=\Psi_{0}
\end{aligned}
$$


and

$$
\begin{aligned}
\varphi_{4} & =\Psi_{4}+\frac{1}{d+2} \eta \Psi_{2}+\frac{1}{d(d-2)} \eta^{2} \Psi_{0} \\
D & =\frac{1}{2}\left[\Psi_{4}^{\prime}+\frac{2}{d+2} \Psi_{2}+\frac{1}{d+2} \eta \Psi_{2}^{\prime}+\frac{2}{d(d-2)} \eta \Psi_{0}\right] .
\end{aligned}
$$

At this point we have managed to construct fields $\Psi_{n}$ which satisfy the Fronsdal off-shell condition $\Psi_{n}^{\prime \prime}=0$ but this is not enough. We should check that the decomposition in (3.10), or equivalently (3.15), decompose the Lagrangian (3.3) into a series of Fronsdal Lagrangians for the irreducible fields $\Psi_{n}$. We demonstrate this explicitly for the $\mathrm{s}=4$ case in Appendix $\mathrm{A}$, where we show that all cross-terms between $\Psi_{n}, n=0,2,4$ vanish.

spin-s The above arguments draw a clear strategy for finding the decomposition for the general spin-s $\|$ We assume an expansion of the form

$$
\Psi_{s}=\sum_{q=0}^{\left[\frac{s}{2}\right]} \rho_{q}(d-2, s) \eta^{q} \mathcal{W}_{s}^{q}
$$

Imposing the double tracelessness condition it turns out that we get more equations than parameters since the traces of $\mathcal{W}$ are not written in terms of $\mathcal{W}$ only (see 3.12).

So the system seems over-constrained since we have to demand that both the coefficients of $\varphi^{[q]}$ terms and $\mathcal{W}^{q}$ vanish after taking the double trace. Nevertheless, surprisingly, we find a solution

$$
\rho_{q}(d-2, s)=-\frac{\rho_{q-1}(d-2, s)}{(d+2(s-q-2))}=\frac{(-1)^{q}(d+2(s-q-3)) ! !}{(d+2(s-3)) ! !}
$$

which appeared in [13]. In the same manner we can show that in general

$$
\Psi_{s-2 k}=\sum_{q=0}^{\left[\frac{s}{2}\right]-k} \rho_{q}(d-2, s-2 k) \eta^{q} \mathcal{W}_{s}^{q+k}
$$

are doubly traceless fields. Taking the gauge transformation of this equation we can show, using $\rho_{k}(d-2, s)=\rho_{k}(d, s-1)$, that $\delta \Psi_{s-2 k}=\partial \tilde{\Lambda}_{s-2 k}$ with the traceless gauge parameter

$$
\tilde{\Lambda}_{s-1-2 k}=\sum_{q=0}^{\left[\frac{s}{2}\right]} \rho_{q}(d, s-2 k-1) \eta^{q} \Lambda_{s-1}^{[q+k]}
$$

\footnotetext{
"It should be clear that the basis we have chosen for the decomposition in (3.16) is not the largest possible we could have constructed. In principle the independent "basis-vectors" we could have written are $\varphi, D$ and all their traces, a total of $s+1$ terms. We have used instead only the $\left[\frac{s}{2}\right]+1$ linear combinations of them given by $\mathcal{W}_{s}^{q}$. This is motivated by our observation that the gauge transformations of $\mathcal{W}_{s}^{q}$ have the proper form for giving irreducible fields gauge transformation with traceless gauge parameters. It is plausible that our basis might not be unique.
} 
If one tries to invert these equations one gets a system of $\left[\frac{s}{2}\right]$ linear equations with a lower diagonal matrix. We write an expansion of the form

$$
\mathcal{W}_{s}^{q}=\sum_{k=0}^{\left[\frac{s}{2}\right]-q} \tilde{\rho}_{k}(d, s-2 q) \eta^{k} \Psi_{s-2 q-2 k}
$$

Generalizing equations (3.9) and (3.14) for the cases $s=2,4,6$ we make an ansatz

$$
\tilde{\rho}_{k}(d, s)=\frac{(d+2(s-2 k-2)) ! !}{(d+2(s-k-2)) ! !} .
$$

To verify our ansatz we insert (3.20) in (3.16) and vice versa. Then i.e. inserting the expansion of $\mathcal{W}_{s}^{q}$ into the expansion of $\Psi_{s}$ we should get a Kroenecker delta on the RHS of (3.16) as it is required by consistency with the LHS. The same for the other way around. We finally get the condition

$\delta_{0, u}=\sum_{n=0}^{u}\left(\begin{array}{l}u \\ n\end{array}\right) \tilde{\rho}_{n}(d, h) \rho_{u-n}(d-2, h-2 n)=\sum_{n=0}^{u}\left(\begin{array}{l}u \\ n\end{array}\right) \tilde{\rho}_{u-n} n(d, h-2 n) \rho_{n}(d-2, h)$.

A direct computation with Mathematica gives a non-vanishing result only for $u=0$, which implies the validity of the ansatz (3.21) as a solution of (3.20) for arbitrary spin $s$. We have also checked it by hand up to spin 6 . Further on we need the expansion of $\varphi, D$ in terms of $\Psi$ in order to verify that the irreducible modes decouple among each other and to determine the normalization of the kinetic term for each HS irreducible mode. These are given by

$$
\begin{aligned}
\varphi & =\mathcal{W}^{0}=\sum_{k=0}^{\left[\frac{s}{2}\right]} \tilde{\rho}_{k}(d, s) \eta^{k} \Psi_{s-2 k} \\
D=\frac{1}{2}\left(\left(\mathcal{W}^{0}\right)^{\prime}-\mathcal{W}^{1}\right) & =\frac{1}{2} \sum_{k=0}^{\left[\frac{s}{2}\right]-1} \tilde{\rho}_{k}(d, s) \eta^{k} \Psi_{s-2 k}^{\prime}+\sum_{k=1}^{\left[\frac{s}{2}\right]} \tilde{\rho}_{k}(d, s) \eta^{k-1} \Psi_{s-2 k} \cdot(3
\end{aligned}
$$

Now we can insert these expressions in the action (3.3) and verify that all crossterms vanish and therefore the fields decouple. We have done this only up to spin 4 (see Appendix A) but we are confident that it works for all spins. In any case taking as a fact the decoupling, the next thing to do is to compute the normalization of the Fronsdal Lagrangian for each irreducible higher spin field as it appears in the original Lagrangian (3.2) after we insert the decomposition (3.23). For this computation it is more convenient to use (3.3). The key point is that we should look for the normalization of the $\left(\partial_{\mu} \Psi_{n}\right)^{2}$ term for each irreducible field. The standard normalization in Fronsdal Lagrangian (3.6) is $-\frac{1}{2}$. In this manner we get from (B.6) the normalization for the propagator (the inverse of the prefactor of $\left(\partial_{\mu} \Psi_{s-2 k}\right)^{2}$ terms multiplied by 2)

$$
Q(s, k, d)=\frac{2^{k} k !(s-2 k) !}{s ! \tilde{\rho}_{k}(d, s)}
$$




\subsection{The Current-Current Interaction}

In this subsection we will rewrite the interaction Lagrangian (2.25) in terms of the irreducible HS fields $\Psi_{n}$. We will be interested in an interaction term which contains a single $\mathcal{W}_{s}^{q}$. The resulting Lagrangian term after we insert (3.20) is

$$
\mathcal{L}(s, h)=\mathcal{W}_{s}^{q} \cdot J_{s-2 q}=\sum_{k=0}^{\left[\frac{s}{2}\right]-q} \tilde{\rho}_{k}(d, s-2 q)\left(\eta^{k} \Psi_{s-2 q-2 k}\right) \cdot J_{s-2 q}
$$

where $h=s-2 q$. Using the identity $(\bar{B} .2)$ and

$$
\eta^{k} \cdot J_{s-2 q}=(2 k-1) ! ! J_{s-2 q}^{[k]}
$$

we get

$$
\mathcal{L}(s, h)=\sum_{k=0}^{\left[\frac{s}{2}\right]-q} \tilde{\rho}_{k}(d, s-2 q) \frac{h !}{2^{k} k !(h-2 k) !} \Psi_{h-2 k} \cdot J_{h}^{[k]} .
$$

The Lagrangian can be written as

$$
\mathcal{L}(s, h)=\sum_{k=0}^{h} \tilde{J}_{h-2 k} \cdot \Psi_{h-2 k}
$$

with

$$
\tilde{J}_{h-2 k}=\tilde{\rho}_{k}(d, h) \frac{h !}{2^{k} k !(h-2 k) !} J_{h}^{[k]} .
$$

The currents $\tilde{J}_{h-2 k}$ are conserved since $J_{h}$ are conserved for on-shell scalar fields in (2.27). As explained in [13] we can compute the current-current interaction between those currents using a projector of the form** $\mathcal{P}^{h-2 k}$, although the currents are not doubly traceless as one would naively expect. It can be shown that despite this apparent paradox, the correct number of physical modes is exchanged when we compute the current-current interaction for conserved currents just as in our case. Actually we can see that, if we try to construct double traceless currents from the currents of (2.27) exactly as in (3.16), we get a current which is not traceless conserved (traceless conserved current means that its double trace and the traceless part of the divergence vanish separately) as it is required by gauge invariance of the total, free plus interaction, Lagrangian $\dagger$.

** The operator $\mathcal{P}$ is polynomial of powers of $\eta_{\mu \nu}$ and $\Pi_{\mu \nu}=\eta_{\mu \nu}-p_{\mu} \bar{p}_{\nu}-p_{\nu} \bar{p}_{\mu}$ where $p^{2}=$ $\bar{p}^{2}=0, p \cdot \bar{p}=1$, which guarantees that only physical degrees of freedom are exchanged between external currents. When acting on conserved currents the expression of $\mathcal{P}$ simplifies and becomes completely independent of $\bar{p}_{\mu}$, see (3.32).

${ }^{\dagger \dagger}$ Indeed gauge variation of the free plus interacting Lagrangian leads to the condition

$$
\delta \mathcal{L}=\int \Lambda \partial \cdot J=0
$$


The expression for the current exchange of two conserved currents coupled to the irreducible higher spin fields $\Psi_{h-2 k}$ is given by

$$
\tilde{J}_{h-2 k} \cdot \mathcal{P}^{h-2 k} \cdot \tilde{J}_{h-2 k}=\sum_{n=0}^{\left[\frac{h}{2}\right]-k} \rho_{n}(d-2, h-2 k) \frac{(h-2 k) !}{2^{n} n !(h-2 k-2 n) !} \tilde{J}_{h-2 k}^{[n]} \cdot \tilde{J}_{h-2 k}^{[n]}
$$

including the propagator normalization (3.24) and using the expressions of $\tilde{J}_{h-2 k}$ from (3.29) we can write the current exchange for the $\mathcal{W}_{q}^{s}$ field (after a shift $k+n=u$ )

$$
\begin{aligned}
\mathcal{A}(s, h)= & (h !)^{2} \sum_{k=0}^{h} \frac{(s-h+2 k) ! !}{s !} \frac{\tilde{\rho}_{k}(d, h)^{2}}{2^{2 k}(k !)^{2} \tilde{\rho}_{k+\left[\frac{s-h}{2}\right]}(d, s)} . \\
& \sum_{u=k}^{\left[\frac{h}{2}\right]} \frac{\rho_{u-k}(d-2, h-2 k)}{2^{u-k}(u-k) !(h-2 u) !} J_{h}^{[u]} \cdot J_{h}^{[u]} .
\end{aligned}
$$

A change in the order of the summations leads to the expression

$$
\mathcal{A}(s, h)=\frac{h !^{2}}{s !} \sum_{u=0}^{\left[\frac{h}{2}\right]} \frac{J_{h}^{[u]} \cdot J_{h}^{[u]}}{2^{u}(h-2 u) !} \sum_{k=0}^{u} \frac{(s-h+2 k) ! !}{2^{k}(k !)^{2}(u-k) !} \frac{\tilde{\rho}_{k}(d, h)^{2}}{\tilde{\rho}_{k+\left[\frac{s-h}{2}\right]}(d, s)} \rho_{u-k}(d-2, h-2 k) .
$$

This is our final expression for the current exchanges written in terms of irreducible fields. This expression has some remarkable properties. In particular, taking $h=s$ and using (3.22) we get the extremely simple expression

$$
A(s, s)=J_{s} \cdot J_{s} .
$$

We see that all traces of the currents have cancelled. We shall see in the next section that this is exactly the result which is obtained after using the propagator of the $\mathcal{W}_{s}^{0}=\varphi_{s}$ field.

What is even more remarkable is the fact that in the above result the only nonvanishing contributions are those where $u \leq \max \left(\left[\frac{s-h}{2}\right],\left[\frac{h}{2}\right]\right)$. We have checked this property numerically with the use of Mathematica for several values up to $s=$ 100. This is perfectly consistent with the value one can expect for the form of the propagator for the p-th trace of $\varphi_{s}$ (see (4.12)), which gives further support to our results.

which requires, for a double traceless current $\mathrm{J}$ of spin $\mathrm{s}$, that

$$
\partial \cdot J=\frac{1}{d+2(s-3)} \eta\left(\partial \cdot J^{\prime}\right)
$$

since the gauge parameter is traceless for the gauge variation of an irreducible field. 


\section{Current Exchanges For Triplets}

In this section we will repeat the computation performed in the previous Section using a different method. We will gauge fix the Lagrangian in (2.22) in a specific gauge in which the fields $\varphi, D$ decouple from each other and we shall write down the propagator for those fields in this gauge. Computing the current-current interaction we will confirm that our result (3.34) has the correct form and the remarkable constrain on the current traces mentioned after (3.35) appears naturally in perturbation theory formulated in terms of reducible fields. We will also demonstrate the equivalence of the two methods with several non-trivial examples.

\subsection{Gauge Fixing And The Propagator}

The most straightforward gauge is the one where the auxiliary field $C_{s-1}$ in (2.22) is set equal to zero. The gauge fixing ( $R_{\xi}$-gauge) term in the Lagrangian has the form

$$
\mathcal{L}_{\xi}=-\frac{1}{2 \xi} C^{2}
$$

The gauge fixing procedure requires the introduction of a Faddeev-Popov determinant in the path integral

$$
Z=\int d \omega e^{-\frac{\omega^{2}}{2 \xi}} \int[d \varphi][d C][d D] \Delta_{F P} \delta(C-\omega) e^{\mathcal{L}+\mathcal{L}_{\xi}}
$$

where using the gauge transformation of $C$ from (2.24) one gets

$$
\Delta_{F P}=\operatorname{det}\left(\frac{\delta C}{\delta \Lambda}\right)=\operatorname{det}(\square)
$$

The FP determinant is field independent and can be absorbed into the normalization constant of the path integral. Obviously, the presence of interactions in the Lagrangian will make the FP determinant field dependent, requiring therefore that we introduce ghosts just as in QCD. In our case though the interaction (2.25) is abelian since it gauges the abelian rigid symmetries of the free scalar Lagrangian (see i.e., [1] and [19]) and ghost fields will not be needed. On the contrary, when we consider the full interacting Lagrangian as i.e. (2.16)-(2.21), we will need FP ghost fields for a consistent quantum theory. This, however, will not affect tree level amplitudes in full analogy with QCD, since external states are always on-shell, but it will play an important role in loop amplitudes and in the optical theorem. We leave this and other interesting issues for a future work, where it would be very interesting to consider a consistent interacting theory beyond the tree level.

The value of the parameter $\xi$ interpolates from Dedonder-Feynman gauge for $\xi=0$ to Dedonder-Landau for $\xi=1 \$$. The most useful gauge for our purposes is

\footnotetext{
$\ddagger$ Notice that in the literature for QED the $R_{\xi}$ gauge fixing Lagrangian is given by $\mathcal{L}_{\xi}=$
} 
the Feynman gauge which basically decouples the field $C$ completely from the path integral and the gauge fixed Lagrangian takes the form

$$
\mathcal{L}+\mathcal{L}_{\xi=0}=-\frac{1}{2}\left(\partial_{\mu} \varphi\right)^{2}+\frac{s(s-1)}{2}\left(\partial_{\mu} D\right)^{2} .
$$

It is instructive to use the equations of motion for $C$ from (2.23) to deduce the form of the gauge fixing in terms of irreducible fields. Inserting (3.23) in the second equation of (2.23) we get

$$
C=\partial \cdot \varphi-\partial D=\sum_{k=0}^{\left[\frac{s}{2}\right]-1} \tilde{\rho}_{k}(d, s) \eta^{k}\left(\partial \cdot \Psi_{s-2 k}-\frac{1}{2} \partial \Psi_{s-2 k}^{\prime}\right)
$$

where in the RHS we recognize immediately the Dedonder gauge fixing term in the parentheses. The Feynman gauge fixing corresponds to setting $C=0$ in the Lagrangian.

We notice from (4.4) that the two fields $\varphi, D$ have decoupled completely from each other, allowing us to simply invert their kinetic operators in order to get their propagators

$$
\begin{aligned}
\Delta(\varphi ; \mu, \varphi ; \nu) & =\frac{\eta_{\mu_{1}\left(\nu_{1}\right.} \eta_{\mu_{2} \nu_{2}} \ldots \eta_{\left.\mu_{s} \nu_{s}\right)}}{p^{2} s !} \\
\Delta(D ; \mu, D ; \nu) & =-\frac{1}{s(s-1)} \frac{\eta_{\mu_{1}\left(\nu_{1}\right.} \eta_{\mu_{2} \nu_{2}} \ldots \eta_{\left.\mu_{s-2} \nu_{s-2}\right)}}{p^{2}(s-2) !} \\
\Delta(\varphi ; \mu, D)=0 &
\end{aligned}
$$

where the parentheses in subscripts signify symmetrization with respect to one set of the indices i.e., $\nu_{1}, \ldots \nu_{s}$. Notice the negative sign in the propagator of $D$. The field $D$ is a "ghost" field. For other gauges like the Landau one, the fields $\varphi, D$ do not decouple after integrating out the auxiliary field $C$ and it is quite non-trivial to diagonalize the kinetic operator to get the propagator of these states.

Let us see how this procedure works explicitly for the spin 2 case. The propagators for the irreducible fields $\Psi_{2}, \Psi_{0}$ from (3.8) in the Feynman gauge are

$$
\begin{aligned}
\Delta\left(\Psi_{2} ; \mu, \Psi_{2} ; \nu\right) & =\frac{\eta_{\mu_{1} \nu_{1}} \eta_{\mu_{2} \nu_{2}}+\eta_{\mu_{1} \nu_{2}} \eta_{\mu_{2} \nu_{1}}-\frac{2}{d-2} \eta_{\mu_{1} \mu_{2}} \eta_{\nu_{1} \nu_{2}}}{2 p^{2}} \\
\Delta\left(\Psi_{0}, \Psi_{0}\right) & =\frac{d-2}{p^{2}}
\end{aligned}
$$

\footnotetext{
$-\frac{1}{2 \xi}\left(\partial_{\mu} A^{\mu}\right)$ and the Feynman gauge corresponds to $\xi=1$. This corresponds to adding (4.1) to (2.22) for the spin-1 triplet, integrating out $C$ first, which gives $C=\partial_{\mu} A^{\mu}$, and then gauge fixing with a $\delta(\partial \cdot A-\omega)$ condition in the path integral. This procedure gives us for $\xi=1$ the Feynman gauge and for $\xi=0$ the Landau one. This is exactly the opposite identification from the main text where gauge fixing $C$ as in (4.2), rather than integrating it out, we get Landau gauge for $\xi=1$ and Feynman gauge for $\xi=0$.
} 
where we have taken into account the normalization factors of the kinetic term of $\Psi_{0}$. Now using equations (3.9) we can deduce the propagators for the fields $\varphi=\Psi_{2}+\frac{1}{d+2} \eta \Psi_{0}$ and $D=\frac{1}{2} \Psi_{2}^{\prime}+\frac{1}{d-2} \Psi_{0}$

$$
\begin{aligned}
\Delta(\varphi ; \mu, \varphi ; \nu) & =\frac{\eta_{\mu_{1} \nu_{1}} \eta_{\mu_{2} \nu_{2}}+\eta_{\mu_{1} \nu_{2}} \eta_{\mu_{2} \nu_{1}}}{2 p^{2}} \\
\Delta(D, D) & =-\frac{1}{2 p^{2}} \\
\Delta(\varphi ; \mu, D)=0 &
\end{aligned}
$$

where we easily see that the fields $\varphi, D$ are decoupled from each other and the propagators agree with (4.6). The fact that this decoupling is special to the Feynman gauge can be seen if we repeat the procedure described above in Landau gauge where the propagators take the form

$$
\begin{aligned}
\Delta\left(\Psi_{2} ; \mu, \Psi_{2} ; \nu\right)= & \frac{\eta_{\mu_{1} \nu_{1}} \eta_{\mu_{2} \nu_{2}}+\eta_{\mu_{1} \nu_{2}} \eta_{\mu_{2} \nu_{1}}}{2 p^{2}} \\
& -\frac{\frac{2 \eta_{\mu_{1} \mu_{2}} \eta_{\nu_{1} \nu_{2}}}{d-2}+\frac{\eta_{\mu_{1} \nu_{1}} p_{\mu_{2}} p_{\nu_{2}}+3 \text { permutations }}{p^{2}}}{2 p^{2}} \\
\Delta\left(\Psi_{0}, \Psi_{0}\right)= & \frac{d-2}{p^{2}} .
\end{aligned}
$$

From the form of the propagators above we can easily compute the propagator $\Delta(\varphi ; \mu, D)$ and indeed we find that it is non-zero and therefore the fields do not decouple.

\subsection{Current Exchange and Comparison}

In this section we will compute the current-current interaction for the Lagrangian (3.25) using the propagators in (4.6). For this we will need to compute the propagators for arbitrary traces of the fields $\Delta\left(\varphi^{[p]}, \varphi^{[p]}\right)$. There are obviously propagators of the form $\Delta\left(\varphi^{[p]}, \varphi^{[q]}\right)$ for $p \neq q$ but they will not be needed for the current exchange computations we consider in this note. From the form of the propagators in (4.6) and the use of (3.5) we can deduce the general form of these propagators

$$
\Delta\left(\varphi_{s}^{[p]} ; 1, \varphi_{s}^{[p]} ; 2\right)=\sum_{k=\max \left(2 p-\left[\frac{s}{2}\right]\right)}^{p} B_{k}(s, p) \eta_{1}^{p-k} \eta_{2}^{p-k} \Delta\left(\varphi_{s-4 p+2 k}^{[p]} ; 1, \varphi_{s-4 p+2 k}^{[p]} ; 2\right)
$$

where $p \in\left[0,\left[\frac{s}{2}\right]\right]$. The notation needs some explanation. The two sets of indices for the two fields of the propagators are denoted in a shorthand notation in which, 1 stands for the $\mu_{1}, \mu_{2}, \ldots \mu_{s-2 p}$ subscripts of the first field and 2 for the $\nu_{1}, \nu_{2}, \ldots \nu_{s-2 p}$ subscripts of the second field. The propagators in the summation on the RHS are the usual propagators of (4.6) for the field with spin $s-4 p-2 k$. The coefficients 
$B_{k}(s, p)$ are unknowns to be determined by the explicit calculation. This has not been achieved for the moment in the general case. The lower bound in the k-summation is obvious since the minimum spin of the propagators in the RHS is zero.

Now, an important observation is that each $\eta_{1}^{p-k} \eta_{2}^{p-k}$ term will give us a currentcurrent interaction proportional to $J_{s}^{p-k} \cdot J_{s}^{p-k}$. The lower bound of the summation means that the maximum trace of the currents allowed in a $\varphi_{s}^{[p]}$ exchange can be written as

$$
\max \left(\left[\frac{s}{2}\right]-p, p\right)=\max \left(\frac{s-h}{2},\left[\frac{h}{2}\right]\right)
$$

We immediately recognize the bound we deduced numerically from (3.34). The equation (4.13) explains the surprising constrain which we pointed out in the last paragraph of the Section 3 . This fact strongly indicates that the procedures described in Section 3 and Section 4 are completely equivalent. This was not obvious at all in the expression of (3.34) but it is a direct consequence of our $\Delta\left(\varphi_{s}^{[p]} ; 1, \varphi_{s}^{[p]} ; 2\right)$ propagators in the theory formulated in terms of $\varphi$ and $D$.

Since we have not achieved to the moment to compute the explicit expressions of the $B_{k}(s, p)$ coefficients we will proceed with a few examples which establish the equivalence of the two methods we have used. Let us define

$$
C(s-h, u, h) \equiv \sum_{k=0}^{u} \frac{(s-h+2 k) ! !}{2^{k} k !^{2}(u-k) !} \frac{\tilde{\rho}_{k}(d, h)^{2}}{\tilde{\rho}_{k+\left[\frac{s-h}{2}\right]}(d, s)} \rho_{u-k}(d-2, h-2 k)
$$

which appears in (3.34). Numerical computation of the coefficients $C(s-h, u, h)$ gives zero for $u>\frac{s-h}{2}$.

Spins 2 and 4 We can easily compute the relevant coefficients $C(s-h, u, h)$ we will need for the spin 2 and 4 cases

$$
\begin{array}{cl}
C(2,0, h)=2(d+2(h-1)) & C(2,1, h)=2 \\
C(4,0, h)=8(d+2 h-2)(d+2 h) & C(4,1, h)=16(d+2 h-2) \\
& C(4,2, h)=4 .
\end{array}
$$

From the explicit expression of the propagators in (4.6), the interaction Lagrangian (2.25) and the definition of $\mathcal{W}_{s}^{q}$ in (2.26) we get for the corresponding current exchanges

$$
\begin{aligned}
& \mathcal{A}(2,2)=p^{2} J_{2} \Delta\left(W_{2}^{0}, W_{2}^{0}\right) J_{2}=J_{2} \cdot J_{2} \\
& \mathcal{A}(2,0)=p^{2} J_{0} \Delta\left(W_{2}^{1}, W_{2}^{1}\right) J_{0}=(d-2) J_{0} \cdot J_{0}
\end{aligned}
$$

where $\mathcal{A}(s, h)$ is the current exchange for the $\mathcal{W}_{q}^{s}$ field as in (3.34). The current exchange is defined as the residue of the corresponding Feynman diagram for the current-current interaction. We can compare (4.16) now with the expression from (3.34) using (4.15). We verify that $\mathcal{A}(2,0)=\frac{C(2,0,0)}{2} J_{0} \cdot J_{0}$ while $\mathcal{A}(2,2)$ is given by (3.35) as expected. 
The spin 4 case requires that we compute the propagators of traces of fields. The relevant propagators, after a short computation, are given by

$$
\begin{aligned}
\Delta\left(\varphi^{\prime} ; \mu, \varphi^{\prime} ; \nu\right) & =2 \frac{(d+4) \eta_{\mu_{1}\left(\nu_{1}\right.} \eta_{\left.\mu_{2} \nu_{2}\right)}+2 \eta_{\mu_{1} \mu_{2}} \eta_{\nu_{1} \nu_{2}}}{4 ! p^{2}} \\
\Delta\left(\varphi^{\prime \prime}, \varphi^{\prime \prime}\right) & =8 \frac{d(d+2)}{4 ! p^{2}} \\
\Delta(D ; \mu, D ; \nu) & =-\frac{1}{12} \frac{\eta_{\mu_{1}\left(\nu_{1} 1\right.} \eta_{\left.\mu_{2} \nu_{2}\right)}}{2 ! p^{2}} \\
\Delta\left(D^{\prime}, D^{\prime}\right) & =-\frac{d}{12 p^{2}} .
\end{aligned}
$$

For $h=2$ we get

$$
\mathcal{A}(4,2)=p^{2} J_{2} \Delta\left(\mathcal{W}_{4}^{1}, \mathcal{W}_{4}^{1}\right) J_{2}=\frac{2(d+2) J_{2} \cdot J_{2}+2 J_{2}^{[1]} \cdot J_{2}^{[1]}}{12}
$$

which is reproduced by the expression in (3.34)

$$
\mathcal{A}(4,2)=\frac{(2 !)^{2}}{4 !} \sum_{u=0}^{1} \frac{C(2, u, 2)}{(2-2 u) ! 2^{u}} J_{2}^{[u]} \cdot J_{2}^{[u]} .
$$

In a similar manner we obtain that $\mathcal{A}(4,0)=\frac{8 d(d-2)}{12} J_{0} \cdot J_{0}$. So we have shown that both methods agree for spin 2 and spin 4 .

Spin $\mathbf{s}$ and $\mathbf{h}=\mathbf{s}-\mathbf{2}$ case For this case we need to compute the propagator $\Delta\left(\varphi^{\prime} ; 1, \varphi^{\prime} ; 2\right)$. Keeping a more compact notation we find

$$
\Delta\left(\varphi^{\prime}, \varphi^{\prime}\right)=\frac{2}{p^{2}}\left(\frac{d+2(s-2)}{s(s-1)} \Delta_{s-2}(1,2)+2 \frac{\eta_{1} \eta_{2} \Delta_{s-4}(1,2)}{s(s-1)(s-2) s-3)}\right) .
$$

The subscripts of $\Delta$ on the RHS are the spin of the propagator from (4.6) and we have suppressed the field variables and their space-time indices. The second term on the RHS implies symmetrization of each $\eta_{i}, i=1,2$ with the corresponding indices of the $\Delta_{s-4}(1,2)$ propagator. When the propagator is contracted with symmetric currents $J_{s-2}$ for both sets of indices then there are $\frac{(s-2)(s-3)}{2}$ terms from the symmetrization of each $\eta_{i}$ which result into $\frac{(s-2)(s-3)}{2}$ traces of the currents $J_{s-2}^{[1]}$ for each set of indices. The final result we get is

$$
\begin{aligned}
\mathcal{A}(s, s-2)= & p^{2} J_{s-2} \Delta\left(\mathcal{W}_{s}^{1}, \mathcal{W}_{s}^{1}\right) J_{s-2} \\
& =2 \frac{d+2(s-3)}{s(s-1)} J_{s-2} \cdot J_{s-2}+\frac{(s-2)(s-3)}{s(s-1)} J_{s-2}^{[1]} \cdot J_{s-2}^{[1]} .
\end{aligned}
$$

The same computation should be reproduced by (3.34)

$$
\mathcal{A}(s, s-2)=\frac{(s-2) !^{2}}{s !} \sum_{u=0}^{\left[\frac{s}{2}\right]-1} \frac{C(2, u, s-2)}{(s-2-2 u) ! 2^{u}} J_{s-2}^{[u]} \cdot J_{s-2}^{[u]} .
$$


Taking into account the constraint (4.13) we see that only $u=0,1$ survive and a trivial computation using (4.14) gives agreement of the two results.

Further examples become more and more tedious. Nevertheless, we believe that we have demonstrated in a sufficient manner the equivalence of the two methods. Actually our result in (3.34) can be used to extract the $B_{k}(s, p)$ coefficients rather than trying to compute them directly by taking traces of (4.6).

\section{Conclusions}

In this paper we developed a technique of constructing propagators for massless irreducible higher spin modes from the Lagrangians describing the reducible higher spin fields. The main motivation for this is that often the gauge-invariant Lagrangians describing the reducible higher spin modes have a much simpler form than those describing irreducible higher spin modes, especially when one considers interacting theories. This technique can be straightforwardly generalized to the case of massive higher spin fields. As an application we considered the current-current exchange amplitudes obtained from the cubic Lagrangians describing an interaction of higher spin fields with scalars.

It would be interesting to generalise these results for the case of $A d S$ space and for the triplets containing fermionic fields. Another interesting application of our results may be a computation of the higher order scattering amplitudes for systems which contain exact vertexes in all orders in the coupling constant. A possible example of such kind of systems is given in the present paper for massive and in [14] for massless bosonic higher spin fields.

\section{Acknowledgements}

We are grateful to C.Angelantonj and A. Sagnotti for useful discussions. We would also like to thank the referee for several interesting points we have clarified and correcting erroneous factors in a formula. The work of A. F. was supported by an INFN postdoctoral fellowship'and partly supported by the Italian MIUR-PRIN contract 20075ATT78. The work of M.T. was supported by a STFC rolling grant $\mathrm{ST} / \mathrm{G} 00062 \mathrm{X} / 1$.

\section{A Appendix A: Decoupling of the Lagrangian for Spin 2 and Spin 4 triplet}

The Lagrangian for spin $\mathrm{s}=4$ in $(\underline{3.3})$ is given by

$$
\begin{aligned}
\mathcal{L}= & -\frac{1}{10}\left(\partial_{(k} \varphi_{\mu \nu \rho \sigma)}\right)^{2}+4\left(\left(\partial \cdot \varphi_{\nu \rho \sigma}\right)\right)^{2}-4\left(\partial \cdot \varphi_{\nu \rho \sigma}\right) D^{\nu \rho \sigma} \\
& +4\left(\partial_{(k} D_{\mu \nu)}\right)^{2}-12\left(\left(\partial \cdot D_{\mu}\right)\right)^{2}
\end{aligned}
$$


We insert the decomposition (3.15) and using (3.5) we get from each of the five terms of the Lagrangian (A.1) the cross-terms listed in Table 1.

\begin{tabular}{|c|c|c|c|c|c|c|}
\hline$M . T$ & $-\frac{1}{10}(\partial \varphi)^{2}$ & $+4(\partial \cdot \varphi)^{2}$ & $-4(\partial \cdot \varphi) D$ & $+4(\partial D)^{2}$ & $-12(\partial \cdot D)^{2}$ & Total \\
\hline$\frac{\left(\partial \Psi_{4}^{\prime}\right)\left(\partial \Psi_{2}\right)}{d+2}$ & -2 & 0 & -2 & 4 & 0 & 0 \\
\hline$\frac{\left(\partial \cdot \Psi_{4}\right)\left(\partial \Psi_{2}\right)}{d+2}$ & -4 & 8 & -4 & 0 & 0 & 0 \\
\hline$\frac{\left(\partial \cdot \Psi_{4}^{\prime}\right)\left(\partial \cdot \Psi_{2}\right)}{d+2}$ & 0 & 24 & -12 & 0 & -12 & 0 \\
\hline$\frac{\left(\partial \cdot \Psi_{4}^{\prime}\right)\left(\partial \Psi_{2}^{\prime}\right)}{d+2}$ & 0 & 0 & -6 & 12 & -6 & 0 \\
\hline$\frac{\left(\partial \cdot \Psi_{4}^{\prime}\right)\left(\partial \Psi_{0}\right)}{d(d-2)}$ & -12 & 24 & -24 & 24 & -12 & 0 \\
\hline$\frac{\left(\partial \cdot \Psi_{2}\right)\left(\partial \Psi_{0}\right)}{d(d+2)(d-2)}$ & $-12(d+4)$ & $24(d+4)$ & $-12(d+6)$ & +48 & -24 & 0 \\
\hline$\frac{\left(\partial \Psi_{2}^{\prime}\right)\left(\partial \Psi_{0}\right)}{d(d+2)(d-2)}$ & $-6(d+4)$ & 24 & $-6(d+6)$ & $12(d+4)$ & -12 & 0 \\
\hline
\end{tabular}

Table 1

In table 1 the numbers in each column indicate the coefficient of the cross terms of $\Psi_{n}$ (first column) from each term of the Lagrangian (A.1) (first row). "M.T" stands for "Mixing Terms".

As we can see all mixing terms have vanishing coefficients and we confirm that the Lagrangian decomposes to gauge invariant Lagrangians for $\Psi_{4}, \Psi_{2}, \Psi_{0}$. Since the fields $\Psi_{n}$ are by definition doubly traceless and transform with traceless parameters (3.19), we can deduce easily that the Lagrangians for the irreducible fields are Fronsdal Lagrangians. We are only missing the correct normalization coefficient for each one of them. This will be computed in Appendix B for the general spin-s case. A few examples of our calculations from the first term in the Lagrangian are

$$
\begin{aligned}
\left(\partial \Psi_{4}\right)\left(\eta \partial \Psi_{2}\right) & =10\left[\partial \Psi_{4}^{\prime}+2\left(\partial \cdot \Psi_{4}\right)\right]\left(\partial \Psi_{2}\right) \\
\left(\eta \partial \Psi_{2}\right)^{2} & =10\left[(d+6)\left(\partial \Psi_{2}\right)^{2}+12\left(\partial \cdot \Psi_{2}\right)^{2}+3\left(\partial \Psi_{2}^{\prime}\right)^{2}+12\left(\partial \cdot \Psi_{2}\right)\left(\partial \Psi_{2}^{\prime}\right)\right] \\
\left(\partial \Psi_{4}\right)\left(\eta^{2} \partial \Psi_{0}\right) & =60\left(\partial \cdot \Psi_{4}^{\prime}\right)\left(\partial \Psi_{0}\right) \\
\left(\eta \partial \Psi_{2}\right)\left(\eta^{2} \partial \Psi_{0}\right) & =30(d+4)\left(2\left(\partial \cdot \Psi_{2}\right)+\partial \Psi_{2}^{\prime}\right)\left(\partial \Psi_{0}\right)
\end{aligned}
$$

Let us explain a bit how we get the result of the first equation in (A.2). The expression on the LHS has total 10 terms. These come from the symmetrization of $\eta$ with $\partial \Psi_{2}$. Then depending which terms $\eta$ contracts from $\partial \Psi_{4}$ we get $\eta_{\mu \nu} \partial^{\mu} \Psi_{4}^{\nu \rho \sigma \lambda}$ or $\eta_{\mu \nu} \partial^{\rho} \Psi_{4}^{\mu \nu \sigma \lambda}$. In this way we obtain the two terms on the RHS with the given multiplicities.

\section{B Appendix B: Lagrangian Normalization for Ir- reducible Fields}

In this appendix we will prove equation (3.24) of the main text. 
Using (3.5) and various manipulations we have the following identities

$$
\begin{aligned}
\left(\eta^{k} \partial \Psi_{s-2 k}\right)^{2} & =\frac{(s+1) !}{\tilde{\rho}_{k}(d, s+2) 2^{k} k !(s-2 k+1) !}\left(\partial \Psi_{s-2 k}\right)^{2}+\ldots \\
\left(\partial \cdot\left(\eta^{k} \Psi_{s-2 k}\right)\right)^{2} & =\frac{(s-1) !}{\tilde{\rho}_{k-1}(d, s) 2^{k-1}(k-1) !(s-2 k+1) !}\left(\partial \Psi_{s-2 k}\right)^{2}+\ldots \\
\left(\eta^{k-2} \partial \Psi_{s-2 k}\right)^{2} & =\frac{(s-3) !}{\tilde{\rho}_{k-2}(d, s-2) 2^{k-2}(k-2) !(s-2 k+1) !}\left(\partial \Psi_{s-2 k}\right)^{2}+\ldots
\end{aligned}
$$

where the dots are all terms involving traces and divergences of the fields $\Psi_{s-2 k}$ and we have used the following combinatorial identity for symmetrized tensors contracted with symmetrized tensors

$$
\eta^{k} T_{q} \rightarrow \eta^{k} \times T_{q} \frac{(2 k+q) !}{(2 k) ! q !}
$$

- Notice that $(\overline{B .2})$ is not an equality. What it means is that if the tensor of the LHS is contracted with a tensor totally symmetric over all the indices then we can substitute it with the expression on the RHS. The symbol $\times$ means the product of the two tensors without symmetrization of their indices. We also remind the reader that as in [28] the notation $\eta^{k}$ has a total of $(2 k-1)$ !! terms unlike the symmetrization of a $2 k$-rank tensor which has $(2 k)$ ! terms.

Using the identities above we can insert (3.23) in (3.3) dropping the cross-terms and keeping only the $\left(\partial \Psi_{s-2 k}\right)^{2}$ terms which are relevant to our case. We get

$$
\begin{aligned}
& \frac{s !}{2^{k+1} k !(s-2 k+1) !} \frac{\tilde{\rho}_{k}^{2}(d, s)}{\tilde{\rho}_{k-1}(d, s)} \cdot \\
& \left(d-2(s-k)+\frac{4(k-1) k}{d+2(s-k-1)}-4 k\right)\left(\partial \Psi_{s-2 k}\right)^{2}+\ldots
\end{aligned}
$$

where we have used the identities

$$
\begin{aligned}
\tilde{\rho}_{k}(d, s+2) & =\frac{\tilde{\rho}_{k-1}(d, s)}{d+2(s-k)} \\
\tilde{\rho}_{k-1}(d, s) & =\frac{\tilde{\rho}_{k-2}(d, s-2)}{d+2(s-k-1)} .
\end{aligned}
$$

Now we can use the following identity (which was used also to convert (3.2) to (3.3))

$$
\left(\partial_{\mu} \Psi_{q}\right)^{2}=\frac{1}{q+1}(\partial \Psi)^{2}-q\left(\partial \cdot \Psi_{q}\right)^{2}
$$

to arrive finally at

$$
\mathcal{L}_{\varphi, D} \rightarrow-\frac{1}{2} \tilde{\rho}_{k}(d, s)^{2} \frac{s !}{2^{k} k !(s-2 k) !}\left(\partial_{\mu} \Psi_{s-2 k}\right)^{2}
$$


after we have used the identity

$$
\tilde{\rho}_{k}(d, s)=\tilde{\rho}_{k-1}(d, s) \frac{(d+2(s-2 k))(d+2(s-2 k-1)}{d+2(s-k-1))} .
$$

(B.6) should be contrasted to the coefficient of the first term in (3.6).

\section{References}

[1] A. Fotopoulos, N. Irges, A. C. Petkou and M. Tsulaia, JHEP 0710 (2007) 021 arXiv:0708.1399 [hep-th]].

[2] X. Bekaert, S. Cnockaert, C. Iazeolla and M. A. Vasiliev, arXiv:hep-th/0503128. N. Bouatta, G. Compere and A. Sagnotti, arXiv:hep-th/0409068. D. Sorokin, AIP Conf. Proc. 767, 172 (2005) |arXiv:hep-th/0405069].

[3] A. Fotopoulos and M. Tsulaia, Int. J. Mod. Phys. A 24, 1 (2009) arXiv:0805.1346 [hep-th]].

[4] E. S. Fradkin and M. A. Vasiliev, Nucl. Phys. B 291, 141 (1987). E. S. Fradkin and M. A. Vasiliev, Phys. Lett. B 189 (1987) 89. M. A. Vasiliev, arXiv:hep-th/9910096. M. A. Vasiliev, Phys. Lett. B 243, 378 (1990). M. A. Vasiliev, Phys. Lett. B 285, 225 (1992). M. A. Vasiliev, Phys. Lett. B 567, 139 (2003) arXiv:hep-th/0304049. O. A. Gelfond, E. D. Skvortsov and M. A. Vasiliev, Theor. Math. Phys. 154, 294 (2008) [arXiv:hep-th/0601106]. K. B. Alkalaev and M. A. Vasiliev, Nucl. Phys. B 655, 57 (2003) arXiv:hep-th/0206068.

[5] C. Fronsdal, Phys. Rev. D 18 (1978) 3624.

[6] A. K. H. Bengtsson, I. Bengtsson and L. Brink, Nucl. Phys. B 227, 31 (1983). A. K. H. Bengtsson, I. Bengtsson and L. Brink, Nucl. Phys. B 227, 41 (1983). F. A. Berends, G. J. H. Burgers and H. van Dam, Nucl. Phys. B 260, 295 (1985). I. G. Koh and S. Ouvry, Phys. Lett. B 179, 115 (1986) [Erratum-ibid. 183B, 434(E) (1987)]

[7] F. A. Berends, G. J. H. Burgers and H. van Dam, Nucl. Phys. B 271, 429 (1986).

[8] A. K. H. Bengtsson, Class. Quant. Grav. 5, 437 (1988).

[9] D. Anselmi, Class. Quant. Grav. 17, 1383 (2000) arXiv:hep-th/9906167].

[10] R. R. Metsaev, Nucl. Phys. B 759, 147 (2006) arXiv:hep-th/0512342. R. R. Metsaev, arXiv:0712.3526 [hep-th]. 
[11] X. Bekaert, N. Boulanger and S. Cnockaert, JHEP 0601, 052 (2006) arXiv:hep-th/0508048. N. Boulanger and S. Leclercq, JHEP 0611, 034 (2006) arXiv:hep-th/0609221.

[12] I. L. Buchbinder, A. Fotopoulos, A. C. Petkou and M. Tsulaia, Phys. Rev. D 74, 105018 (2006) arXiv:hep-th/0609082.

[13] D. Francia, J. Mourad and A. Sagnotti, Nucl. Phys. B 773, 203 (2007) arXiv:hep-th/0701163].

[14] A. Fotopoulos and M. Tsulaia, Phys. Rev. D 76 (2007) 025014 arXiv:0705.2939 [hep-th]].

[15] G. Bonelli, Nucl. Phys. B 669 (2003) 159 arXiv:hep-th/0305155]. N. Moeller and P. C. West, Nucl. Phys. B 729 (2005) 1 arXiv:hep-th/0507152.

[16] D. Francia, J. Mourad and A. Sagnotti, Nucl. Phys. B 804, 383 (2008) arXiv:0803.3832 [hep-th]].

[17] Yu. M. Zinoviev, Class. Quant. Grav. 26, 035022 (2009) arXiv:0805.2226 [hepth]].

[18] N. Boulanger, S. Leclercq and P. Sundell, JHEP 0808, 056 (2008) arXiv:0805.2764 [hep-th]].

[19] X. Bekaert, E. Joung and J. Mourad, JHEP 0905, 126 (2009) arXiv:0903.3338 [hep-th]].

[20] R. Manvelyan, K. Mkrtchyan and W. Ruhl, arXiv:0903.0243 [hep-th]. R. Manvelyan and K. Mkrtchyan, arXiv:0903.0058 [hep-th].

[21] A. K. H. Bengtsson, J. Math. Phys. 48 (2007) 072302 [arXiv:hep-th/0611067].

[22] D. Francia and A. Sagnotti, Phys. Lett. B 543, 303 (2002) arXiv:hep-th/0207002]. D. Francia and A. Sagnotti, Phys. Lett. B 624, 93 (2005) |arXiv:hep-th/0507144]. D. Francia, Nucl. Phys. B 796, 77 (2008) arXiv:0710.5378 [hep-th]]. A. Campoleoni, D. Francia, J. Mourad and A. Sagnotti, arXiv:0904.4447 [hep-th]. A. Campoleoni, D. Francia, J. Mourad and A. Sagnotti, Nucl. Phys. B 815, 289 (2009) arXiv:0810.4350 [hep-th]].

[23] I. A. Bandos and J. Lukierski, Mod. Phys. Lett. A 14, 1257 (1999) arXiv:hep-th/9811022. I. A. Bandos, J. Lukierski and D. P. Sorokin, Phys. Rev. D 61, 045002 (2000) arXiv:hep-th/9904109.

[24] R. R. Metsaev, Phys. Lett. B 671, 128 (2009) [arXiv:0808.3945 [hep-th]]. R. R. Metsaev, arXiv:0907.2207 [hep-th]. R. R. Metsaev, Phys. Rev. D 78, 106010 (2008) arXiv:0805.3472 [hep-th]]. 
[25] P. A. Horvathy, M. S. Plyushchay and M. Valenzuela, Phys. Rev. D 77 (2008) 025017 [arXiv:0710.1394 [hep-th]].

[26] F. Bastianelli and R. Bonezzi, JHEP 0903, 063 (2009) arXiv:0901.2311 [hep-th]]. F. Bastianelli, O. Corradini and E. Latini, JHEP 0811, 054 (2008) arXiv:0810.0188 [hep-th]]. A. R. Gover, A. Shaukat and A. Waldron, arXiv:0812.3364 [hep-th]. R. Marnelius, arXiv:0906.2084 [hep-th].

[27] S. Ouvry and J. Stern, Phys. Lett. B 177, 335 (1986). A. K. H. Bengtsson, Phys. Lett. B 182, 321 (1986). A. K. H. Bengtsson, Nucl. Phys. B 333 (1990) 407.

[28] D. Francia and A. Sagnotti, Class. Quant. Grav. 20 (2003) S473 arXiv:hep-th/0212185].

[29] A. Fotopoulos, K. L. Panigrahi and M. Tsulaia, Phys. Rev. D 74, 085029 (2006) arXiv:hep-th/0607248].

[30] D. P. Sorokin and M. A. Vasiliev, Nucl. Phys. B 809, 110 (2009) arXiv:0807.0206 [hep-th]].

[31] A. Sagnotti and M. Tsulaia, Nucl. Phys. $\quad$ B 682 (2004) 83 arXiv:hep-th/0311257].

[32] F. Hussain, G. Thompson and P. D. Jarvis, Phys. Lett. B 216, 139 (1989).

[33] C. Burdik, A. Pashnev and M. Tsulaia, Nucl. Phys. Proc. Suppl. 102, 285 (2001) arXiv:hep-th/0103143]. X. Bekaert, I. L. Buchbinder, A. Pashnev and M. Tsulaia, Class. Quant. Grav. 21, S1457 (2004) arXiv:hep-th/0312252].

[34] A. Pashnev and M. M. Tsulaia, Mod. Phys. Lett. A 12, 861 (1997) arXiv:hep-th/9703010].

[35] A. Pashnev and M. Tsulaia, Mod. Phys. Lett. A 13, 1853 (1998) arXiv:hep-th/9803207]. I. L. Buchbinder, A. Pashnev and M. Tsulaia, Phys. Lett. B 523, 338 (2001) arXiv:hep-th/0109067]. I. L. Buchbinder, V. A. Krykhtin and A. Pashnev, Nucl. Phys. B 711, 367 (2005) arXiv:hep-th/0410215.

[36] I. L. Buchbinder and V. A. Krykhtin, Nucl. Phys. B 727, 537 (2005) arXiv:hep-th/0505092]. I. L. Buchbinder, V. A. Krykhtin, L. L. Ryskina and H. Takata, Phys. Lett. B 641, 386 (2006) arXiv:hep-th/0603212. I. L. Buchbinder, V. A. Krykhtin and P. M. Lavrov, Nucl. Phys. B 762, 344 (2007) arXiv:hep-th/0608005]. I. L. Buchbinder, A. V. Galajinsky and V. A. Krykhtin, Nucl. Phys. B 779, 155 (2007) arXiv:hep-th/0702161|. I. L. Buchbinder, V. A. Krykhtin and A. A. Reshetnyak, Nucl. Phys. B 787, 211 (2007) arXiv:hep-th/0703049]. I. L. Buchbinder and A. V. Galajinsky, JHEP 0811, 
081 (2008) arXiv:0810.2852 [hep-th]]. I. L. Buchbinder, V. A. Krykhtin and L. L. Ryskina, Nucl. Phys. B 819, 453 (2009) [arXiv:0902.1471 [hep-th]].

[37] D. J. Gross and A. Jevicki, Nucl. Phys. B 283, 1 (1987).

D. J. Gross and A. Jevicki, Nucl. Phys. B 287, 225 (1987).

A. Neveu and P. C. West, Nucl. Phys. B 278 (1986) 601. 